$57^{\text {ème }}$ Congrès de la SFMBCB, 01001 (2011)

DOI: $10.1051 / \mathrm{sfmbcb} / 20115701001$

(c) Owned by the authors, published by EDP Sciences, 2011

\title{
Le cancer : où en sommes-nous ? A la lumière du plan cancer
}

Viguier J

Institut national du Cancer, Boulogne Billancourt, France

Le bilan à la fin 2010 de la mise en œuvre du Plan cancer 2009-2013 met en évidence un avancement des mesures globalement conforme au calendrier prévu, grâce à l'engagement de tous les acteurs de la recherche et de la lutte contre le cancer et à la mobilisation des ressources programmées.

\section{Construire les soins de demain par le soutien à la recherche, à l'innovation et à la formation des professionnels}

Le soutien à la recherche translationnelle et à la recherche clinique a été renforcé pour un transfert plus rapide des découvertes scientifiques au bénéfice des malades, par le financement de projets de recherche (61 projets ont été sélectionnés en 2010 sur les programmes PHRC et STIC cancer pour un financement total de 22 millions d'euros; 16 projets de recherche translationnelle ont été retenus en 2010 pour 9,2 millions d'euros).

La participation des patients aux essais cliniques a fortement progressé $(+27 \%$ de 2008 à 2009 , passant de 21.700 à 27.700 patients inclus); les efforts seront poursuivis pour atteindre l'objectif fixé par le Plan d'augmenter de 50\% la participation aux essais d'ici 2013.

Les efforts ont été accrus pour faire progresser l'accès aux traitements innovants et aux nouvelles thérapies, par le développement des tests de biologie moléculaire permettant d'identifier les patients répondant aux indications (plus de 10.000 tests pour le cancer colorectal et 15.000 tests pour le cancer du poumon réalisés en 2010) et par la labellisation et le soutien de 16 centres d'essais cliniques de phase précoce.

La participation de la France aux programmes de recherche internationaux s'est développée, en particulier avec le National Cancer Institute américain (NCI-CTEP), et dans le cadre du programme « International Cancer Genome Consortium » (ICGC).

Afin de garantir la qualité et la sécurité des soins dans les années à venir, il est impératif de faire face aux défis démographiques des professionnels de santé en cancérologie, notamment par une progression des effectifs en formation. 886 nouveaux postes d'internes sont ainsi programmés sur la période 2010-2014, répartis en 577 postes en oncologie (92 en 2010) et 309 en anatomopathologie (53 en 2010).

\section{Réduire les inégalités de santé face au cancer par l'amélioration de l'observation, de la prévention et du dépistage}

L'incidence des cancers en France, comme la mortalité par cancer, présente d'importantes disparités géographiques et sociales. Le rapport «la situation du cancer en France », actualisé chaque année, rassemble les données disponibles portant sur l'épidémiologie et les disparités géographiques.

Les efforts sont accrus pour caractériser les risques environnementaux et comportementaux et les inégalités face au cancer. Dans le cadre du Programme national de recherche en environnement santé travail, l'appel à projets 2010 de l'ANSES a été conduit en association avec l'INCa (13 projets sur le cancer sélectionnés). 
La prévention doit participer à la réduction des inégalités face au cancer. Au moins un tiers des cancers seraient évitables, le principal facteur de risque étant le tabac. Pour améliorer l'aide à distance au sevrage tabagique, le programme de coaching personnalisé mis en place par l'INPES sur le site tabac-info-service.fr s'est déployé en 2010.

Il existe des disparités sur le territoire et de fortes inégalités socio-économiques et culturelles dans le recours au dépistage des cancers. Pour améliorer la participation de l'ensemble de la population au dépistage, des actions spécifiques sont déployées en direction de publics cibles, à travers les messages des campagnes d'information et des interventions ciblées en direction des personnes les plus fragiles pour des raisons sociales, culturelles ou économiques. Les professionnels de santé sont également sensibilisés au dépistage et à la détection précoce des cancers, au moyen d'outils d'information adaptés aux pratiques de chacun et de modules de formation.

Afin de permettre la définition de nouvelles stratégies de dépistage, les recommandations sur le dépistage du cancer du col de l'utérus ont été publiées par la HAS. Des recommandations sur le dépistage du cancer de la prostate chez les sujets à risques sont en cours d'élaboration.

\section{Personnaliser les parcours des patients en impliquant les médecins traitants et améliorer la qualité de vie pendant et après le cancer}

La qualité des prises en charge des malades est soutenue par la production de guides et de recommandations. 5 recommandations de prise en charge spécialisée ont été élaborées avec les sociétés savantes et publiées.

Pour rendre accessible aux patients une information de référence sur les cancers, afin de leur permettre de devenir acteurs du système de soins, la plateforme Cancer info mise en place avec la Ligue nationale contre le cancer repose sur un site internet, une ligne téléphonique et des guides thématiques.

Les actions ont été engagées pour développer une prise en charge personnalisée et accompagner l'après cancer, en renforçant le rôle du médecin traitant. Le cadre national du dossier communicant de cancérologie, au sein du dossier médical partagé, est publié (ASIP-INCa) et sera testé dans 7 régions pilotes en 2011.

Les premiers sites de recherche intégrée sur le cancer (SIRIC) devraient être labellisés et soutenus, à l'issue du processus d'évaluation.

Les 885 établissements de santé ayant reçu une autorisation pour le traitement des cancers doivent d'ici mai 2011 remplir tous les critères attendus de sécurité et de qualité en chirurgie, chimiothérapie et radiothérapie.

Le Portail des données du cancer sera ouvert au premier semestre 2011.

La place de la France dans les partenariats internationaux va se renforcer. Le partenariat avec le National Cancer Institute américain devrait être renforcé afin de développer les coopérations et les échanges de pratiques.

\section{La réduction des inégalités de santé face au cancer constitue un objectif transversal du Plan cancer.}

Pour favoriser l'arrêt du tabac, le remboursement des substituts nicotiniques sera triplé à partir de 2011 pour les femmes enceintes et les bénéficiaires de la couverture maladie universelle.

De nouveaux projets de recherche interventionnelle seront soutenus en 2011 pour évaluer les actions menées visant à lutter contre les inégalités de santé contre le cancer. 


\section{L'amélioration de la qualité de vie pendant et après le cancer est une priorité.}

Aujourd'hui un patient sur deux est en vie cinq ans après le diagnostic de cancer ${ }^{1}$. Une mobilisation forte de tous les acteurs impliqués est néanmoins nécessaire

Une nouvelle campagne de communication sur l'image sociale du cancer sera lancée à partir de mai 2011, pour faire changer le regard sur le cancer et sur les personnes touchées par la maladie, en valorisant les progrès de la recherche au bénéfice des malades.

Pour améliorer l'accès aux assurances et au crédit des personnes présentant un risque aggravé de santé, la nouvelle convention AERAS, signée le $1^{\text {er }}$ février 2011 à l'issue des négociations menées avec les représentants des banques et assurances, sera mise en œuvre à partir de 2011.

Une action sera conduite permettant d'améliorer la prise en compte des situations de handicap ou de perte d'autonomie liées au cancer. Cette démarche s'inscrira dans le cadre de l'expérimentation des parcours personnalisés de soins et de l'après-cancer.

S'agissant des actions prévues par le Plan cancer pour lever les obstacles à la réinsertion professionnelle des personnes atteintes de cancer, les stratégies de mise en œuvre vont être définies pour assurer leur réalisation à l'échéance de la durée du Plan

Correspondance : jviguier@institutcancer.fr 